\title{
Case Series of Multisystem Inflammatory Syndrome in Adults Associated with SARS-CoV-2 Infection - United Kingdom and United States, March-August 2020
}

\begin{abstract}
Sapna Bamrah Morris, MD ${ }^{1}$; Noah G. Schwartz, MD ${ }^{1,2}$; Pragna Patel, MD ${ }^{1}$; Lilian Abbo, MD 3 ; Laura Beauchamps, MD ${ }^{3}$; Shuba Balan, MD 3 ;

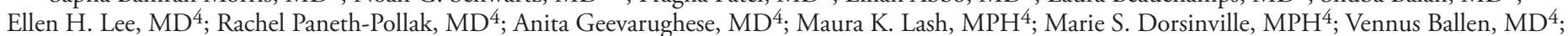
Daniel P. Eiras, $\mathrm{MD}^{4}$; Christopher Newton-Cheh, MD ${ }^{5,6}$; Emer Smith, MPH ${ }^{7,8}$; Sara Robinson, $\mathrm{MPH}^{7}$; Patricia Stogsdill, MD ; Sarah Lim, MBBCh ${ }^{10}$; Sharon E. Fox, MD, PhD 11,12; Gillian Richardson, $\mathrm{MPH}^{13}$; Julie Hand, MSPH ${ }^{13}$; Nora T. Oliver, MD ${ }^{14}$; Aaron Kofman, MD ${ }^{15}$; Bobbi Bryant, $\mathrm{MPH}^{1,16}$; Zachary Ende, $\mathrm{PhD}^{1,16}$; Deblina Datta, MD ${ }^{1}$; Ermias Belay, MD ${ }^{1}$; Shana Godfred-Cato, $\mathrm{DO}^{1}$
\end{abstract}

\section{On October 2, 2020, this report was posted as an MMWR Early Release on the MMWR website (https://www.cdc.gov/mmwr).}

During the course of the coronavirus disease 2019 (COVID-19) pandemic, reports of a new multisystem inflammatory syndrome in children (MIS-C) have been increasing in Europe and the United States (1-3). Clinical features in children have varied but predominantly include shock, cardiac dysfunction, abdominal pain, and elevated inflammatory markers, including C-reactive protein (CRP), ferritin, D-dimer, and interleukin-6 (1). Since June 2020, several case reports have described a similar syndrome in adults; this review describes in detail nine patients reported to CDC, seven from published case reports, and summarizes the findings in 11 patients described in three case series in peer-reviewed journals (4-6). These 27 patients had cardiovascular, gastrointestinal, dermatologic, and neurologic symptoms without severe respiratory illness and concurrently received positive test results for SARS-CoV-2, the virus that causes COVID-19, by polymerase chain reaction (PCR) or antibody assays indicating recent infection. Reports of these patients highlight the recognition of an illness referred to here as multisystem inflammatory syndrome in adults (MIS-A), the heterogeneity of clinical signs and symptoms, and the role for antibody testing in identifying similar cases among adults. Clinicians and health departments should consider MIS-A in adults with compatible signs and symptoms. These patients might not have positive SARS-CoV-2 PCR or antigen test results, and antibody testing might be needed to confirm previous SARS-CoV-2 infection. Because of the temporal association between MIS-A and SARS-CoV-2 infections interventions that prevent COVID-19 might prevent MIS-A. Further research is needed to understand the pathogenesis and long-term effects of this newly described condition.

Potential MIS-A patients were identified from several sources: reports from clinicians and health departments, published case reports, and published case series. Clinicians and health departments in the United States voluntarily reported adult patients with suspected MIS-A to CDC using the case report form* developed for MIS-C after a Health Advisory was published on May 14, 2020, calling for reporting of MIS-C cases. The case report form included information on patient demographics, underlying medical conditions, clinical findings, complications, laboratory test results including those from SARS-CoV-2 testing, imaging findings, treatments, and outcomes. Two clinician reviewers selected patients who fulfilled the working MIS-A case definition used in this report, which included the following five criteria: 1) a severe illness requiring hospitalization in a person aged $\geq 21$ years; 2 ) a positive test result for current or previous SARS-CoV-2 infection (nucleic acid, antigen, or antibody) during admission or in the previous 12 weeks; 3) severe dysfunction of one or more extrapulmonary organ systems (e.g., hypotension or shock, cardiac dysfunction, arterial or venous thrombosis or thromboembolism, or acute liver injury); 4) laboratory evidence of severe inflammation (e.g., elevated CRP, ferritin, D-dimer, or interleukin-6); and 5) absence of severe respiratory illness (to exclude patients in which inflammation and organ dysfunction might be attributable simply to tissue hypoxia). Patients with mild respiratory symptoms who met these criteria were included. Patients were excluded if alternative diagnoses such as bacterial sepsis were identified.

To identify potential published cases, a literature search was performed on August 20, 2020, and 355 publications were identified. ${ }^{\dagger}$ Abstracts were screened by one reviewer to determine whether cases met the working MIS-A case definition; when no abstract was available, the full paper was examined. The references were reviewed to identify additional relevant articles. Data were obtained from published reports; authors were contacted to confirm published data and, when necessary, to provide data not included in the original articles.

\footnotetext{
${ }^{*}$ Multisystem Inflammatory Syndrome Associated with COVID-19 Case Report Form. https://www.cdc.gov/mis-c/pdfs/hcp/mis-c-form-fillable.pdf.

${ }^{\dagger}$ Medline (OVID), Embase (OVID), CINAHL (EBSCOHost) and Cochrane Library were searched as primary sources, which were supplemented with searches in the following databases: Global Health, CAB abstracts, PsycInfo, Scopus, PubMed Central, Global Index Medicus, and several preprint databases. Each database was searched using the following terms: novel coronavirus/ COVID-19 (multiple iterations) and severe inflammation/multisystem, cardiogenic shock/Kawasaki disease, and adult.
} 


\section{Case Reports}

Demographic characteristics and underlying conditions. Cases in nine patients reported to CDC (Table 1) and seven published case reports (Table 2), originating from seven U.S. jurisdictions and the United Kingdom, met the working case definition. The 16 patients ranged in age from 21 to 50 years and included seven men and nine women. Five were reported as Hispanic, nine as African American, one as Asian, and one as a United Kingdom-born man of African ethnicity. Nine patients had no reported underlying medical conditions; six were obese, one had poorly controlled diabetes mellitus type 2 (hemoglobin $\mathrm{A} 1 \mathrm{C}>9.0 \%$ ), two had hypertension, and one had obstructive sleep apnea. Eight patients had documented respiratory illness before developing symptoms of MIS-A, and eight did not.

Initial signs and symptoms. Twelve of 16 patients had fever $\left(\geq 100.4^{\circ} \mathrm{F}\left[38.0^{\circ} \mathrm{C}\right]\right.$ for $\geq 24$ hours or report of subjective fever lasting $\geq 24$ hours) at the time of presentation. Six patients were initially evaluated for possible cardiac symptoms such as chest pain or palpitations; all 16 had evidence of cardiac effects, including electrocardiogram abnormalities such as arrhythmias, elevated troponin levels, or echocardiographic evidence of left or right ventricular dysfunction. Thirteen patients had gastrointestinal symptoms on admission; five had dermatologic manifestations on admission, including three with mucositis. Despite minimal respiratory symptoms, 10 patients had pulmonary ground glass opacities, and six had pleural effusions identified on chest imaging.

Inflammatory markers. All patients had markedly elevated laboratory markers of inflammation, including CRP (range of peak values $=84-580 \mathrm{mg} / \mathrm{L}$; upper limit of normal $[\mathrm{ULN}]=10 \mathrm{mg} / \mathrm{L})$ and ferritin $(196$ to $>100,000 \mathrm{ng} / \mathrm{mL}$; $\mathrm{ULN}=150 \mathrm{ng} / \mathrm{mL}$ for women, $300 \mathrm{ng} / \mathrm{mL}$ for men), as well as markers of coagulopathy including D-dimer $(275-8691 \mathrm{ng} / \mathrm{mL}$; $\mathrm{ULN}=500 \mathrm{ng} / \mathrm{mL}$ ). Ten patients had absolute lymphocyte counts lower than normal range (range of nadir values $120-2120$ cells $/ \mu \mathrm{L}$; lower limit of normal $=1000$ cells $/ \mu \mathrm{L}$ ).

SARS-CoV-2 test results. Ten patients received positive SARS-CoV-2 PCR test results at the time of initial assessment for MIS-A, seven of whom also had serologic evidence of infection (positive antibody test results) at that time. Six patients received negative SARS-CoV-2 PCR test results; of those, four had positive anti-SARS-CoV-2 antibody test results when first evaluated. Two patients had positive SARS-CoV-2 PCR test results 14 and 37 days before admission, negative PCR results at the time of admission, and no known antibody testing. Three additional patients had positive SARS-CoV-2 PCR test results 25-41 days before admission and continued positive PCR test results at the time of admission.
Treatment. Seven patients were treated with intravenous immunoglobulin, 10 with corticosteroids, and two with the interleukin-6 inhibitor, tocilizumab. Ten patients required intensive care; seven required inotropes or vasopressors, and one required mechanical circulatory support (extracorporeal membrane oxygenation followed by temporary left and right ventricular assist devices). Three patients required endotracheal intubation and mechanical ventilation, and two patients died.

\section{Published Case Series}

Three published case series were identified describing adult patients with manifestations consistent with MIS-A (4-6). One series describes seven previously healthy, young adult men aged 20-42 years who experienced mixed cardiogenic and vasoplegic shock and hyperinflammation along with high SARS-CoV-2 immunoglobulin G antibody titers indicating active or previous infection (4). Two of the patients identified as African American, two as Hispanic, two as Middle Eastern, and one as White. Four of the seven patients had negative PCR test results for SARS-CoV-2 at the time of admission, all had markedly elevated inflammatory markers and required inotropes or vasopressors, and three required intraaortic balloon pumps. All were treated with corticosteroids and therapeutic anticoagulation. All seven patients recovered and were discharged home after 7 to 18 days of hospitalization with improved cardiovascular function.

A second case series describes two patients aged 21 and 50 years who came to medical attention because of large-vessel strokes associated with positive SARS-CoV-2 tests (5). Information on race/ethnicity of these patients was not reported. These patients had elevated inflammatory markers and minimal respiratory symptoms, consistent with MIS-A. The authors proposed endothelial dysfunction and coagulopathy related to SARS-CoV-2 infection as potential etiologies. Incidence of large-vessel stroke among young adults during this same time the previous year was statistically significantly lower (5).

A third case series describes the pathologic findings of endothelialitis and complement deposition in the vessels of two patients with illness resembling MIS-A (cardiac dysfunction, abdominal signs and symptoms, and rash) associated with positive SARS-CoV-2 test results (6). Information on race/ ethnicity of these patients was not reported. One of these two patients had no underlying medical conditions and recovered; the other had multiple underlying conditions at higher risk for severe COVID-19 and died hours after seeking care. Pathologic findings in this case series were similar to autopsy findings for those of patient 14 (Table 2). 
TABLE 1. Demographics, clinical features, treatments, and outcomes of nine adults reported to CDC with multisystem inflammatory syndrome (MIS) associated with SARS-CoV-2 infection - United States, March-August 2020

\begin{tabular}{|c|c|c|c|c|c|c|c|c|}
\hline $\begin{array}{l}\text { Age (yrs), sex, } \\
\text { race/ethnicity, } \\
\text { location }\end{array}$ & $\begin{array}{l}\text { Underlying } \\
\text { medical } \\
\text { conditions }\end{array}$ & $\begin{array}{l}\text { Clinical signs and } \\
\text { symptoms }\end{array}$ & $\begin{array}{l}\text { Previous } \\
\text { respiratory } \\
\text { illness/ } \\
\text { SARS-CoV-2 } \\
\text { testing }\end{array}$ & $\begin{array}{l}\text { SARS-CoV-2 } \\
\text { testing at time of } \\
\text { MIS-A admission }\end{array}$ & $\begin{array}{l}\text { Laboratory studies } \\
\text { (peak)* }\end{array}$ & $\begin{array}{l}\text { Imaging/Other } \\
\text { diagnostic studies }\end{array}$ & Treatments & $\begin{array}{l}\text { Outcome and } \\
\text { length of } \\
\text { stay }\end{array}$ \\
\hline $\begin{array}{l}\text { Patient 1: } \\
\text { 27, female, } \\
\text { African } \\
\text { American, } \\
\text { Maine }\end{array}$ & None & $\begin{array}{l}\text { Rigors, profuse } \\
\text { diarrhea, diffuse } \\
\text { rash } \times 5 \text { days. } \\
\text { Admitted with } \\
\text { mixed shock } \\
\text { (hypovolemic, } \\
\text { vasoplegic, } \\
\text { cardiogenic) and } \\
\text { acute renal failure. }\end{array}$ & $\begin{array}{l}\text { No/Testing } \\
\text { unknown }\end{array}$ & $\mathrm{PCR}(-), \mathrm{Ab}(+)$ & $\begin{array}{l}\text { CRP } 344 \mathrm{mg} / \mathrm{L} ; \\
\text { D-dimer } 2818 \mathrm{ng} / \mathrm{mL} ; \\
\text { ferritin } 1082 \mathrm{ng} / \mathrm{mL} ; \\
\text { troponin I } 0.43 \mathrm{ng} / \mathrm{mL} ; \\
\text { ALT } 37 \mathrm{IU} / \mathrm{L} ; \\
\text { ALC nadir } 420 \text { cells } / \mu \mathrm{L}\end{array}$ & $\begin{array}{l}\text { TTE: mild to moderate global } \\
\text { hypokinesis, left ventricular } \\
\text { ejection fraction } 45 \% \text {, mildly } \\
\text { dilated right ventricle, mild } \\
\text { tricuspid regurgitation, } \\
\text { pericardial effusion. } \\
\text { CT chest: bilateral patchy } \\
\text { ground-glass opacities, pleural } \\
\text { effusion. } \\
\text { CT abdomen/pelvis: abdominal } \\
\text { free fluid. }\end{array}$ & $\begin{array}{l}\text { Norepinephrine, } \\
\text { vasopressin, } \\
\text { midodrine, } \\
\text { heparin, } \\
\text { corticosteroids }\end{array}$ & $\begin{array}{l}\text { Discharged } \\
\text { after } \\
13 \text { days }\end{array}$ \\
\hline $\begin{array}{l}\text { Patient 2: } \\
\text { 50, male, } \\
\text { African } \\
\text { American, } \\
\text { Florida }\end{array}$ & None & $\begin{array}{l}\text { Poor oral intake, } \\
\text { chest pressure, } \\
\text { palpitations, } \\
\text { diaphoresis } \times 3 \text { days. } \\
\text { Hemodynamically } \\
\text { unstable on } \\
\text { admission. }\end{array}$ & $\begin{array}{l}\text { No/Testing } \\
\text { unknown }\end{array}$ & $\mathrm{PCR}(+), \mathrm{Ab}(+)$ & $\begin{array}{l}\text { CRP } 84 \mathrm{mg} / \mathrm{L} ; \\
\text { D-dimer } 2310 \mathrm{ng} / \mathrm{mL} ; \\
\text { ferritin } 1919 \mathrm{ng} / \mathrm{mL} ; \\
\text { troponin I } 0.48 \mathrm{ng} / \mathrm{mL} ; \\
\text { ALT } 440 \mathrm{IU} / \mathrm{L} ; \\
\text { ALC nadir } 2500 \text { cells } / \mu \mathrm{L}\end{array}$ & $\begin{array}{l}\text { EKG: atrial fibrillation/flutter with } \\
\text { rapid ventricular response, ST } \\
\text { segment changes. } \\
\text { TTE: ejection fraction } 25 \%-30 \% \\
\text { with global hypokinesis. } \\
\text { CXR: small pleural effusions. }\end{array}$ & $\begin{array}{l}\text { Remdesivir, } \\
\text { corticosteroids }\end{array}$ & $\begin{array}{l}\text { Discharged } \\
\text { after } \\
17 \text { days }\end{array}$ \\
\hline $\begin{array}{l}\text { Patient 3: } \\
\text { 46, male, } \\
\text { African } \\
\text { American, } \\
\text { Florida }\end{array}$ & $\begin{array}{l}\text { Obesity, } \\
\text { chronic } \\
\text { right lower } \\
\text { extremity } \\
\text { pain }\end{array}$ & $\begin{array}{l}\text { Malaise, bilateral } \\
\text { tinnitus, chest pain, } \\
\text { and vomiting } \\
\text { x } 4 \text { days. } \\
\text { Hypotensive and } \\
\text { mildly hypoxemic } \\
\text { on admission. }\end{array}$ & $\begin{array}{l}\text { Yes/Testing } \\
\text { unknown }\end{array}$ & $\operatorname{PCR}(-), \mathrm{Ab}(+)$ & $\begin{array}{l}\text { CRP } 217 \mathrm{mg} / \mathrm{L} ; \\
\text { D-dimer } 3790 \mathrm{ng} / \mathrm{mL} ; \\
\text { ferritin > 100,000 ng/mL; } \\
\text { troponin I } 2.5 \mathrm{ng} / \mathrm{mL} ; \\
\text { IL-6 } 1412 \mathrm{pg} / \mathrm{mL} ; \\
\text { ALT >10,000 IU/L; } \\
\text { ALC nadir } 400 \text { cells } / \mu \mathrm{L}\end{array}$ & $\begin{array}{l}\text { EKG: ST-T segment changes. } \\
\text { CT chest: dependent ground } \\
\text { glass opacities. } \\
\text { CT abdomen: hepatic steatosis. }\end{array}$ & $\begin{array}{l}\text { Vasopressors, } \\
\text { tocilizumab x } 1 \text {, } \\
\text { heparin }\end{array}$ & Deceased \\
\hline $\begin{array}{l}\text { Patient 4: } \\
\text { 21, male, } \\
\text { African } \\
\text { American, } \\
\text { Louisiana }\end{array}$ & Obesity & $\begin{array}{l}\text { Fever, cough, } \\
\text { nausea, vomiting, } \\
\text { lymphadenopathy } \\
\text { x } 6 \text { days. }\end{array}$ & $\begin{array}{l}\text { No/Testing } \\
\text { unknown }\end{array}$ & $\mathrm{PCR}(-), \mathrm{Ab}(+)$ & $\begin{array}{l}\text { CRP } 318 \mathrm{mg} / \mathrm{L} ; \\
\text { D-dimer } 1760 \mathrm{ng} / \mathrm{mL} ; \\
\text { ferritin } 4400 \mathrm{ng} / \mathrm{mL} ; \\
\text { troponin T } 0.65 \mathrm{ng} / \mathrm{mL} ; \\
\text { IL-6 } 7 \mathrm{pg} / \mathrm{mL} ; \\
\text { ATL } 279 \mathrm{IU} / \mathrm{L} ; \\
\text { ALC nadir } 700 \text { cells } / \mu \mathrm{L}\end{array}$ & $\begin{array}{l}\text { TTE: severely decreased ejection } \\
\text { fraction, mild mitral regurgitation, } \\
\text { right ventricular dysfunction, } \\
\text { coronary artery dilatation. } \\
\text { CT chest: ground glass opacities } \\
\text { and atelectasis. }\end{array}$ & $\begin{array}{l}\text { ASA, } \\
\text { corticosteroids, } \\
\text { IVIG } \times 1\end{array}$ & $\begin{array}{l}\text { Discharged } \\
\text { after } 6 \text { days }\end{array}$ \\
\hline $\begin{array}{l}\text { Patient 5: } \\
\text { 33, male, } \\
\text { African } \\
\text { American, } \\
\text { Georgia }\end{array}$ & $\begin{array}{c}\text { Obesity, } \\
\text { HTN, } \\
\text { depression }\end{array}$ & $\begin{array}{l}\text { Fever, chest pain, } \\
\text { abdominal pain, } \\
\text { diarrhea, dark urine } \\
\text { x } 4 \text { days. }\end{array}$ & $\begin{array}{c}\text { Yes/PCR (+) } \\
41 \text { days earlier }\end{array}$ & $\mathrm{PCR}(+), \mathrm{Ab}(+)$ & $\begin{array}{l}\text { CRP } 182 \mathrm{mg} / \mathrm{L} ; \\
\text { D-dimer } 275 \mathrm{ng} / \mathrm{mL} ; \\
\text { ferritin } 375 \mathrm{ng} / \mathrm{mL} ; \\
\text { troponin I } 1.8 \mathrm{ng} / \mathrm{mL} ; \\
\text { IL-6 } 74.3 \mathrm{pg} / \mathrm{mL} ; \\
\text { ALT } 30 \mathrm{IU} / \mathrm{L} ; \\
\text { ALC nadir } 2070 \text { cells } / \mu \mathrm{L}\end{array}$ & $\begin{array}{l}\text { CT chest: atelectasis. } \\
\text { CT abdomen/pelvis: normal. } \\
\text { TTE: mitral and tricuspid } \\
\text { regurgitation. }\end{array}$ & Anticoagulation & $\begin{array}{l}\text { Discharged } \\
\text { after } 5 \text { days }\end{array}$ \\
\hline $\begin{array}{l}\text { Patient 6: } \\
\text { 22, female, } \\
\text { African } \\
\text { American, } \\
\text { New York }\end{array}$ & None & $\begin{array}{l}\text { Fever, chills, throat } \\
\text { pain, odynophagia } \\
\times 2 \text { days. }\end{array}$ & $\begin{array}{l}\text { No/Testing } \\
\text { unknown }\end{array}$ & $\mathrm{PCR}(+), \mathrm{Ab}(+)$ & $\begin{array}{l}\text { CRP } 355 \mathrm{mg} / \mathrm{L} ; \\
\text { D-dimer } 1882 \mathrm{ng} / \mathrm{mL} ; \\
\text { ferritin } 378 \mathrm{ng} / \mathrm{mL} ; \\
\text { troponin T } 0.06 \mathrm{ng} / \mathrm{mL} ; \\
\text { IL-6 } 34.8 \mathrm{pg} / \mathrm{mL} ; \\
\text { ALT } 119 \mathrm{U} / \mathrm{L} ; \\
\text { ALC nadir } 360 \text { cells } / \mu \mathrm{L}\end{array}$ & $\begin{array}{l}\text { CT neck: retropharyngeal and } \\
\text { parapharyngeal edema. } \\
\text { EKG: intermittent complete heart } \\
\text { block with narrow junctional } \\
\text { escape without hemodynamic } \\
\text { compromise. } \\
\text { TTE: ejection fraction } 50 \% \text {. } \\
\text { CXR: dense bilateral lower lobe } \\
\text { air-space disease. }\end{array}$ & $\begin{array}{l}\text { Phenylephrine, } \\
\text { anticoagulation, } \\
\text { corticosteroids }\end{array}$ & $\begin{array}{l}\text { Discharged } \\
\text { after } \\
19 \text { days }\end{array}$ \\
\hline
\end{tabular}

See table footnotes on the next page.

\section{Discussion}

Findings indicate that adult patients of all ages with current or previous SARS-CoV-2 infection can develop a hyperinflammatory syndrome resembling MIS-C. Although hyperinflammation and extrapulmonary organ dysfunction have been described in hospitalized adults with severe COVID-19, these conditions are generally accompanied by respiratory failure (7). In contrast, the patients described here had minimal respiratory symptoms, hypoxemia, or radiographic abnormalities in accordance with the working case definition, which was meant to distinguish MIS-A from severe COVID-19; only eight of 16 patients had any documented respiratory symptoms before onset of MIS-A.

The pathophysiology of MIS in both children and adults is currently unknown. Eight of 27 (30\%) adults described in this report and $45 \%$ of 440 children with MIS-C reported to CDC through July 29, 2020, (1) had negative PCR and positive SARS-CoV-2 antibody test results, suggesting MIS-A and MIS-C might represent postinfectious processes. However, in some patients, persistent infection outside the upper respiratory tract is possible; SARS-CoV-2 has been identified in 
TABLE 1. (Continued) Demographics, clinical features, treatments, and outcomes of nine adults reported to CDC with multisystem inflammatory syndrome (MIS) associated with SARS-CoV-2 infection — United States, March-August 2020

\begin{tabular}{|c|c|c|c|c|c|c|c|c|}
\hline $\begin{array}{l}\text { Age (yrs), sex, } \\
\text { race/ethnicity, } \\
\text { location }\end{array}$ & $\begin{array}{l}\text { Underlying } \\
\text { medical } \\
\text { conditions }\end{array}$ & $\begin{array}{l}\text { Clinical signs and } \\
\text { symptoms }\end{array}$ & $\begin{array}{l}\text { Previous } \\
\text { respiratory } \\
\text { illness/ } \\
\text { SARS-CoV-2 } \\
\text { testing }\end{array}$ & $\begin{array}{c}\text { SARS-CoV-2 } \\
\text { testing at time of } \\
\text { MIS-A admission }\end{array}$ & $\begin{array}{l}\text { Laboratory studies } \\
\text { (peak)* }\end{array}$ & $\begin{array}{l}\text { Imaging/Other } \\
\text { diagnostic studies }\end{array}$ & Treatments & $\begin{array}{l}\text { Outcome and } \\
\text { length of } \\
\text { stay }\end{array}$ \\
\hline $\begin{array}{l}\text { Patient 7: } \\
\text { 21, female, } \\
\text { African } \\
\text { American, } \\
\text { New York }\end{array}$ & Obesity & $\begin{array}{l}\text { Fever, fatigue, throat } \\
\text { and neck pain, } \\
\text { nausea, vomiting } \\
\text { x } 1 \text { day. }\end{array}$ & $\begin{array}{c}\text { Yes/PCR (+) } \\
25 \text { days earlier }\end{array}$ & $\mathrm{PCR}(+), \mathrm{Ab}(+)$ & $\begin{array}{l}\text { CRP } 319 \mathrm{mg} / \mathrm{L} ; \\
\text { D-dimer } 713 \mathrm{ng} / \mathrm{mL} ; \\
\text { ferritin } 351 \mathrm{ng} / \mathrm{mL} ; \\
\text { troponin T } 0.04 \mathrm{ng} / \mathrm{mL} ; \\
\text { IL-6 } 56.2 \mathrm{pg} / \mathrm{mL} ; \\
\text { ALT } 160 \mathrm{IU} / \mathrm{L} ; \\
\text { ALC nadir } 260 \text { cells } / \mu \mathrm{L}\end{array}$ & $\begin{array}{l}\text { CT neck: bilateral supraclavicular } \\
\text { and cervical lymphadenopathy } \\
\text { with no discrete abscess or } \\
\text { collection. } \\
\text { CT chest: bilateral patchy } \\
\text { ground-glass opacities, } \\
\text { pleural effusion. } \\
\text { TTE: mild to moderate diffuse left } \\
\text { ventricular hypokinesis. Mild to } \\
\text { moderate decreased left } \\
\text { ventricular ejection fraction (40\%). } \\
\text { Small posterior pericardial } \\
\text { effusion. Mild tricuspid and } \\
\text { mitral valve regurgitation. }\end{array}$ & $\begin{array}{l}\text { Dobutamine, } \\
\text { heparin, ASA x1, } \\
\text { corticosteroids } \times 2\end{array}$ & $\begin{array}{l}\text { Discharged } \\
\text { after } \\
12 \text { days }\end{array}$ \\
\hline $\begin{array}{l}\text { Patient 8: } \\
\text { 47, female, } \\
\text { African } \\
\text { American, } \\
\text { New York }\end{array}$ & None & $\begin{array}{l}\text { Weakness, sore } \\
\text { throat, shortness of } \\
\text { breath, decreased } \\
\text { exercise tolerance } \\
\text { x } 3 \text { days. }\end{array}$ & $\begin{array}{l}\text { Yes/Testing } \\
\text { unknown }\end{array}$ & $\begin{array}{l}\text { PCR (+), } \\
\text { Ab testing } \\
\text { not performed }\end{array}$ & $\begin{array}{l}\text { CRP } 485 \mathrm{mg} / \mathrm{L} ; \\
\text { D-dimer } 1365 \mathrm{ng} / \mathrm{mL} ; \\
\text { ferritin } 948 \mathrm{ng} / \mathrm{mL} ; \\
\text { troponin T } 0.24 \mathrm{ng} / \mathrm{mL} ; \\
\text { ALT } 45 \mathrm{U} / \mathrm{L} ; \\
\text { ALC nadir } 1980 \text { cells } / \mu \mathrm{L}\end{array}$ & $\begin{array}{l}\text { EKG: first degree AV block and } \\
\text { nonspecific T-wave abnormalities. } \\
\text { TTE: borderline left ventricular } \\
\text { ejection fraction ( } 55 \%) \text {. }\end{array}$ & $\begin{array}{l}\text { Heparin, } \\
\text { convalescent } \\
\text { plasma }\end{array}$ & $\begin{array}{l}\text { Discharged } \\
\text { after } 8 \text { days }\end{array}$ \\
\hline $\begin{array}{l}\text { Patient 9: } \\
\text { 42, male, } \\
\text { Asian, New } \\
\text { York }\end{array}$ & Obesity & $\begin{array}{l}\text { Fever, shortness of } \\
\text { breath, cough, } \\
\text { diarrhea, poor } \\
\text { appetite, dysuria } \\
\text { x } 5 \text { days. }\end{array}$ & $\begin{array}{c}\text { Yes/PCR (+) } \\
37 \text { days earlier }\end{array}$ & $\begin{array}{l}\text { PCR (-), } \\
\text { Ab testing } \\
\text { not performed }\end{array}$ & $\begin{array}{l}\text { CRP } 387 \mathrm{mg} / \mathrm{L} ; \\
\text { D-dimer } 3519 \mathrm{ng} / \mathrm{mL} ; \\
\text { ferritin } 7529 \mathrm{ng} / \mathrm{mL} ; \\
\text { troponin T } 0.60 \mathrm{ng} / \mathrm{mL} ; \\
\text { ALT } 66 \mathrm{U} / \mathrm{L} ; \\
\text { ALC nadir } 1740 \text { cells } / \mu \mathrm{L}\end{array}$ & $\begin{array}{l}\text { TEE: mildly dilated left ventricle, } \\
\text { moderately dilated right ventricle, } \\
\text { moderate biventricular } \\
\text { hypokinesis, moderately } \\
\text { decreased left ventricular } \\
\text { ejection fraction ( } 35 \%) \text {. } \\
\text { CXR: bilateral lower lobe opacities/ } \\
\text { airspace disease. }\end{array}$ & $\begin{array}{l}\text { Vasopressors, } \\
\text { anticoagulation, } \\
\text { corticosteroids }\end{array}$ & $\begin{array}{l}\text { Discharged } \\
\text { after } 9 \text { days }\end{array}$ \\
\hline
\end{tabular}

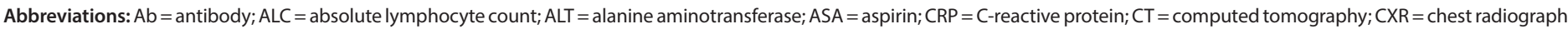

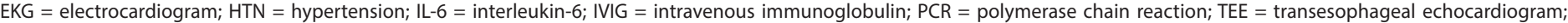
TTE $=$ transthoracic echocardiogram.

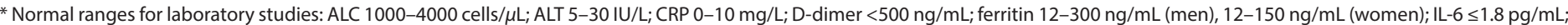
troponin I $<0.03 \mathrm{ng} / \mathrm{mL}$; troponin $\mathrm{T}<0.1 \mathrm{ng} / \mathrm{mL}$.

multiple organs including the heart, liver, brain, kidneys, and gastrointestinal tract (7). Additional proposed mechanisms for extrapulmonary dysfunction in COVID-19 include endothelial damage and thromboinflammation, dysregulated immune responses, and dysregulation of the renin-angiotensinaldosterone system ( 7 ).

The interval between infection and development of MIS-A is unclear, adding to uncertainty regarding whether MIS-A represents a manifestation of acute infection or an entirely postacute phenomenon. In patients with COVID-19, dyspnea is typically experienced a median of 5-8 days and critical illness 10-12 days after onset of symptoms. ${ }^{\S}$ In patients who reported typical COVID-19 symptoms before MIS-A onset, MIS-A was experienced approximately $2-5$ weeks later. However, eight MIS-A patients reported no preceding respiratory symptoms, making it difficult to estimate when initial infection occurred.

Given the high proportion of MIS-C patients with negative PCR testing, clinical guidelines recommend the use of both antibody and viral testing to assist with diagnosis $(8-10)$. In

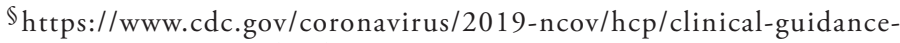
management-patients.html.
}

patients with atypical or late manifestations of SARS-CoV-2 infection, including MIS-A, positive antibody results might be crucial to augment clinical recognition of this condition and guide treatment. In addition, the use of a panel of laboratory tests for inflammation, hypercoagulability, and organ damage (e.g., CRP, ferritin, D-dimer, cardiac enzymes, liver enzymes, and creatinine) might assist in the early identification and management of this COVID-19-associated condition.

All but one of the patients with MIS-A described in this report belonged to racial or ethnic minority groups. Longstanding health and social inequities have resulted in increased risk for infection and severe outcomes from COVID-19 in communities of color. MIS-C has also been reported disproportionately in these communities (1). Because patients described in this review represent a convenience sample from a small number of jurisdictions, conclusions cannot be made regarding the true burden or determinants of MIS-A in different groups; further research is needed.

\footnotetext{
Shttps://www.cdc.gov/coronavirus/2019-ncov/community/health-equity/raceethnicity.html.
} 
TABLE 2. Demographics, clinical features, treatments, and outcomes of seven adults reported in published literature with multisystem inflammatory syndrome (MIS) associated with SARS-CoV-2 infection — United Kingdom and United States, March-August 2020

\begin{tabular}{|c|c|c|c|c|c|c|c|c|}
\hline $\begin{array}{l}\text { Age (yrs), sex, } \\
\text { race/ethnicity, } \\
\text { location }\end{array}$ & $\begin{array}{l}\text { Underlying } \\
\text { medical } \\
\text { conditions }\end{array}$ & Clinical signs/symptoms & $\begin{array}{l}\text { Previous } \\
\text { respiratory } \\
\text { illness/ } \\
\text { SARS-CoV-2 } \\
\text { testing }\end{array}$ & $\begin{array}{l}\text { SARS-CoV-2 } \\
\text { testing at time } \\
\text { of MIS-A } \\
\text { admission }\end{array}$ & $\begin{array}{l}\text { Laboratory studies } \\
\text { (peak)* }\end{array}$ & $\begin{array}{l}\text { Imaging/Other diagnostic } \\
\text { studies }\end{array}$ & Treatments & $\begin{array}{l}\text { Outcome and } \\
\text { length of stay }\end{array}$ \\
\hline $\begin{array}{l}\text { Patient } 10^{\dagger} \text { : } \\
\text { 36, female, } \\
\text { Hispanic, } \\
\text { New York }\end{array}$ & None & $\begin{array}{l}\text { Fever, abdominal pain, } \\
\text { vomiting, and diarrhea } \\
\text { x } 7 \text { days; arthralgias and } \\
\text { diffuse rash x } 2 \text { days. On } \\
\text { admission, nonexudative } \\
\text { conjunctivitis, mucositis, } \\
\text { edema of bilateral hands } \\
\text { and feet, palmar erythema, } \\
\text { diffuse maculopapular rash, } \\
\text { and cervical } \\
\text { lymphadenopathy. }\end{array}$ & No/Not tested & $\operatorname{PCR}(+), \mathrm{Ab}(+)$ & $\begin{array}{l}\text { CRP } 300 \mathrm{mg} / \mathrm{L} ; \\
\text { D-dimer } 652 \mathrm{ng} / \mathrm{mL} ; \\
\text { ferritin } 684 \mathrm{ng} / \mathrm{mL} ; \\
\text { troponin I } 0.07 \mathrm{ng} / \mathrm{mL} ; \\
\text { ALT } 116 \mathrm{IU} / \mathrm{L} ; \\
\text { ALC nadir } 900 \text { cells } / \mu \mathrm{L}\end{array}$ & $\begin{array}{l}\text { TTE: moderate tricuspid } \\
\text { regurgitation, pericardial } \\
\text { effusion. } \\
\text { CT chest: right pleural } \\
\text { effusion. Ultrasound: } \\
\text { gallbladder wall edema. }\end{array}$ & $\begin{array}{l}\text { ASA, IVIG x1, } \\
\text { corticosteroids }\end{array}$ & $\begin{array}{l}\text { Discharged } \\
\text { after } 7 \text { days }\end{array}$ \\
\hline $\begin{array}{l}\text { Patient } 11^{\S} \text { : } \\
\text { 45, male, } \\
\text { Hispanic, } \\
\text { New York }\end{array}$ & None & $\begin{array}{l}\text { Fever, sore throat, diarrhea, } \\
\text { lower extremity pain, and } \\
\text { diffuse rash x } 6 \text { days. On } \\
\text { admission, hypotensive and } \\
\text { tachycardic with } \\
\text { nonexudative conjunctivitis, } \\
\text { periorbital edema, mucositis, } \\
\text { unilateral cervical } \\
\text { lymphadenopathy, and } \\
\text { diffuse exanthem. }\end{array}$ & No/Not tested & $\begin{array}{l}\text { PCR }(+), A b \\
\text { testing not } \\
\text { performed }\end{array}$ & $\begin{array}{l}\text { CRP } 547 \mathrm{mg} / \mathrm{L} ; \\
\text { D-dimer } 2977 \mathrm{ng} / \mathrm{mL} ; \\
\text { ferritin } 21,196 \mathrm{ng} / \mathrm{mL} ; \\
\text { troponin } 8.1 \mathrm{ng} / \mathrm{mL} ; \\
\text { IL-6 } 117 \mathrm{pg} / \mathrm{mL} ; \\
\text { ALT } 133 \mathrm{IU} / \mathrm{L} ; \\
\text { ALC nadir } 700 \text { cells } / \mu \mathrm{L}\end{array}$ & $\begin{array}{l}\text { EKG: ST elevations in } \\
\text { anterolateral leads. } \\
\text { TTE: ejection fraction } 40 \% \\
\text { with global hypokinesis. } \\
\text { CT head/neck: pre-septal } \\
\text { edema. Slit lamp: uveitis. }\end{array}$ & $\begin{array}{l}\text { Heparin, } \\
\text { corticosteroids, } \\
\text { IVIG } \times 2 \text {, } \\
\text { Tocilizumab x } 1\end{array}$ & $\begin{array}{l}\text { Discharged } \\
\text { after } 9 \text { days }\end{array}$ \\
\hline $\begin{array}{l}\text { Patient } 12^{\text {ๆी: }} \\
\text { 44, female, } \\
\text { Hispanic, } \\
\text { Massachusetts }\end{array}$ & $\begin{array}{l}\text { GERD, mild } \\
\text { obstructive } \\
\text { sleep apnea, } \\
\text { depression }\end{array}$ & $\begin{array}{l}\text { Chills, sore throat, cough, } \\
\text { myalgias } x 2 \text { days ( } 8 \text { days } \\
\text { before admission); followed } \\
\text { by diarrhea and back pain } x \\
3 \text { days; followed by pleuritic } \\
\text { chest pain and dyspnea. } \\
\text { Admitted with profound } \\
\text { cardiogenic shock. }\end{array}$ & Yes/Not tested & $\begin{array}{l}\text { PCR }(+), A b \\
\text { testing not } \\
\text { performed }\end{array}$ & $\begin{array}{l}\text { CRP } 141 \mathrm{mg} / \mathrm{L} ; \\
\text { D-dimer } 8691 \mathrm{ng} / \mathrm{mL} ; \\
\text { ferritin } 2564 \mathrm{ng} / \mathrm{mL} ; \\
\text { hs-Trop T } 1810 \mathrm{ng} / \mathrm{L} ; \\
\text { IL-6 } 53.3 \mathrm{pg} / \mathrm{mL} ; \\
\text { ALT } 242 \mathrm{IU} / \mathrm{L} ; \\
\text { ALC nadir } 670 \text { cells } / \mu \mathrm{L}\end{array}$ & $\begin{array}{l}\text { EKG: submillimeter } \\
\text { ST-segment elevation in } \\
\text { leads I/aVL, low QRS voltage. } \\
\text { TTE: severely depressed left } \\
\text { ventricular function, trace } \\
\text { pericardial effusion. } \\
\text { CT chest: mild ground glass } \\
\text { opacities bilateral lung } \\
\text { fields. } \\
\text { CT abdomen/pelvis: small } \\
\text { amount of ascites, } \\
\text { periportal edema. }\end{array}$ & $\begin{array}{l}\text { Norepinephrine, } \\
\text { dobutamine, } \\
\text { vasopressin, } \\
\text { milrinone, IVIG } \\
\text { x } 5 \text { days, ECMO to } \\
\text { LVAD and RVAD. }\end{array}$ & $\begin{array}{l}\text { Discharged to } \\
\text { rehabilitation } \\
\text { facility after } \\
18 \text { days; } \\
\text { home } 7 \text { days } \\
\text { later }\end{array}$ \\
\hline $\begin{array}{l}\text { Patient } 13^{* *} \text { : } \\
21, \text { male, } \\
\text { African } \\
\text { origin, United } \\
\text { Kingdom }\end{array}$ & None & $\begin{array}{l}\text { Fever, headache, and } \\
\text { abdominal pain x } 6 \text { days; } \\
\text { transient palmar rash. } \\
\text { Hypotensive on admission } \\
\text { with nonexudative } \\
\text { conjunctivitis, mucositis, } \\
\text { cervical lymphadenopathy. }\end{array}$ & No/Not tested & $\operatorname{PCR}(-), A b(+)$ & $\begin{array}{l}\text { CRP } 338 \mathrm{mg} / \mathrm{L} ; \\
\text { D-dimer } 4260 \mathrm{ng} / \mathrm{mL} ; \\
\text { ferritin } 1249 \mathrm{ng} / \mathrm{mL} ; \\
\text { troponin T } 3.3 \mathrm{ng} / \mathrm{mL} ; \\
\text { ALT } 330 \mathrm{IU} / \mathrm{L} ; \\
\text { ALC nadir } 390 \text { cells } / \mu \mathrm{L}\end{array}$ & $\begin{array}{l}\text { CT abdomen/pelvis: } \\
\text { mesenteric adenopathy } \\
\text { and ileitis. } \\
\text { EKG: sinus tachycardia. } \\
\text { CT chest: normal. } \\
\text { TTE: normal. } \\
\text { CT coronary angiogram: } \\
\text { normal. }\end{array}$ & $\begin{array}{l}\text { ASA, } \\
\text { corticosteroids, } \\
\text { IVIG x } 1\end{array}$ & $\begin{array}{l}\text { Discharged } \\
\text { after } 8 \text { days }\end{array}$ \\
\hline $\begin{array}{l}\text { Patient } 14^{\dagger+} \text { : } \\
\text { 31, female, } \\
\text { African } \\
\text { American, } \\
\text { Louisiana }\end{array}$ & $\begin{array}{l}\text { Obesity, } \\
\text { HTN, } \\
\text { diabetes } \\
\text { mellitus } \\
\text { type } 2\end{array}$ & $\begin{array}{l}\text { Fever } x 1 \text { day, throbbing neck } \\
\text { pain, nausea, vomiting. }\end{array}$ & $\begin{array}{l}\text { Yes/PCR (+) } \\
14 \text { days before } \\
\text { admission }\end{array}$ & $\begin{array}{l}\text { PCR (-), Ab } \\
\text { testing not } \\
\text { performed }\end{array}$ & $\begin{array}{l}\text { CRP } 580 \mathrm{mg} / \mathrm{L} ; \\
\text { D-dimer } 453 \mathrm{ng} / \mathrm{mL} ; \\
\text { ferritin } 793 \mathrm{ng} / \mathrm{mL} ; \\
\text { ALT } 52 \text { IU/L; } \\
\text { ALC nadir } 2120 \text { cells } / \mu \mathrm{L}\end{array}$ & $\begin{array}{l}\text { Pathology: small-vessel } \\
\text { cardiac vasculitis; new } \\
\text { pulmonary thrombi in a } \\
\text { background of otherwise } \\
\text { reparative changes in the } \\
\text { lungs. } \\
\text { CT head/neck: bilateral } \\
\text { enlarged parotid glands. } \\
\text { CT chest: interval } \\
\text { improvement of bibasilar } \\
\text { ground-glass opacities with } \\
\text { cervical and anterior } \\
\text { mediastinal } \\
\text { lymphadenopathy. }\end{array}$ & CPR & $\begin{array}{l}\text { Deceased at } \\
\text { admission } \\
\text { (ventricular } \\
\text { fibrillation) }\end{array}$ \\
\hline
\end{tabular}

See table footnotes on the next page.

The majority (24 of 27) of patients with MIS-A survived, similar to those with MIS-C, associated with receiving care in acute, often intensive, health care settings. Because of the potential therapies that might benefit these patients as described in these case reports, clinicians should consider MIS-A within a broader differential diagnosis when caring for adult patients with clinical and laboratory findings consistent with the working MIS-A case definition.

The findings in this report are subject to at least three limitations. First, cases described here were voluntarily reported or published and therefore are not representative of the true clinical spectrum or racial/ethnic distribution of this emerging 
TABLE 2. (Continued) Demographics, clinical features, treatments, and outcomes of seven adults reported in published literature with multisystem inflammatory syndrome (MIS) associated with SARS-CoV-2 infection — United Kingdom and United States, March-August 2020

\begin{tabular}{|c|c|c|c|c|c|c|c|c|}
\hline $\begin{array}{l}\text { Age (yrs), sex, } \\
\text { race/ethnicity, } \\
\text { location }\end{array}$ & $\begin{array}{l}\text { Underlying } \\
\text { medical } \\
\text { conditions }\end{array}$ & Clinical signs/symptoms & $\begin{array}{l}\text { Previous } \\
\text { respiratory } \\
\text { illness/ } \\
\text { SARS-CoV-2 } \\
\text { testing }\end{array}$ & $\begin{array}{l}\text { SARS-CoV-2 } \\
\text { testing at time } \\
\text { of MIS-A } \\
\text { admission }\end{array}$ & $\begin{array}{l}\text { Laboratory studies } \\
\text { (peak)* }\end{array}$ & $\begin{array}{l}\text { Imaging/Other diagnostic } \\
\text { studies }\end{array}$ & Treatments & $\begin{array}{l}\text { Outcome and } \\
\text { length of stay }\end{array}$ \\
\hline $\begin{array}{l}\text { Patient } 15^{\S \S} \text { : } \\
25 \text {, female, } \\
\text { Hispanic, } \\
\text { Georgia }\end{array}$ & None & $\begin{array}{l}\text { Fever, weakness, and } \\
\text { shortness of breath } \times 7 \text { days; } \\
\text { followed by sore throat, mild } \\
\text { cough, vomiting, and } \\
\text { diarrhea. Hypotensive on } \\
\text { admission with } \\
\text { conjunctivitis, mucositis, } \\
\text { cervical lymphadenopathy. }\end{array}$ & No/Not tested & PCR (+), Ab (+) & $\begin{array}{l}\text { CRP } 90 \mathrm{mg} / \mathrm{L} ; \\
\text { D-dimer } 1918 \mathrm{ng} / \mathrm{mL} ; \\
\text { ferritin } 798 \mathrm{ng} / \mathrm{mL} ; \\
\text { troponin I } 0.06 \mathrm{ng} / \mathrm{mL} ; \\
\text { ALT } 25 \mathrm{IU} / \mathrm{L} \text {, } \\
\text { ALC nadir } 1150 \text { cells } / \mu \mathrm{L} \\
\end{array}$ & $\begin{array}{l}\text { TTE: moderate to severely } \\
\text { reduced right-sided } \\
\text { ventricular dysfunction, } \\
\text { flattened interventricular } \\
\text { septum in systole consistent } \\
\text { with right ventricular } \\
\text { pressure overload. } \\
\text { EKG: right axis deviation. } \\
\text { CT chest: scattered patchy } \\
\text { ground glass opacities and } \\
\text { peripheral consolidation, } \\
\text { small bilateral pleural } \\
\text { effusions with adjacent } \\
\text { atelectasis; mild } \\
\text { enlargement of the main } \\
\text { pulmonary artery without } \\
\text { pulmonary embolus. } \\
\text { CT abdomen/ pelvis: mild } \\
\text { peripancreatic fat stranding, } \\
\text { nonspecific bilateral } \\
\text { perinephric fat stranding. }\end{array}$ & $\begin{array}{l}\text { ASA, IVIG x 2, } \\
\text { vasopressors }\end{array}$ & $\begin{array}{l}\text { Discharged } \\
\text { after } 5 \text { days }\end{array}$ \\
\hline $\begin{array}{l}\text { Patient 16 } \\
\text { 38, female, } \\
\text { Hispanic, Texas }\end{array}$ & None & $\begin{array}{l}\text { Fever, occipital headache, } \\
\text { conjunctival injection, } \\
\text { odynophagia, mucositis, } \\
\text { glossitis shortness of breath, } \\
\text { vomiting, polyarthralgia, and } \\
\text { rash } \times 5 \text { days. }\end{array}$ & $\begin{array}{l}\text { Yes/PCR (+) } 28 \\
\text { days earlier } \\
\text { d }\end{array}$ & $\mathrm{PCR}(+), \mathrm{Ab}(+)$ & $\begin{array}{l}\text { CRP } 217 \mathrm{mg} / \mathrm{L} ; \\
\text { D-dimer } 1250 \mathrm{ng} / \mathrm{mL} ; \\
\text { ferritin } 196 \mathrm{ng} / \mathrm{mL} ; \\
\text { troponin I }<0.03 \mathrm{ng} / \mathrm{mL} ; \\
\text { ALT } 126 \mathrm{IU} / \mathrm{L} ; \\
\text { ALC nadir } 120 \text { cells } / \mu \mathrm{L}\end{array}$ & $\begin{array}{l}\text { TTE: trace pericardial effusion, } \\
\text { elevated pulmonary artery } \\
\text { pressure (46-51 mmHg), } \\
\text { normal left ventricular } \\
\text { ejection fraction, no } \\
\text { coronary artery } \\
\text { abnormalities. } \\
\text { CT chest/abdomen/pelvis: } \\
\text { no pulmonary emboli, right } \\
\text { upper lobe perihilar } \\
\text { ground-glass opacities, } \\
\text { septal and bronchial wall } \\
\text { thickening, bilateral } \\
\text { small-to-moderate pleural } \\
\text { effusions. }\end{array}$ & $\begin{array}{l}\text { ASA, } \\
\text { corticosteroids, } \\
\text { IVIG x } 2\end{array}$ & $\begin{array}{l}\text { Discharged } \\
\text { after } 7 \text { days }\end{array}$ \\
\hline
\end{tabular}

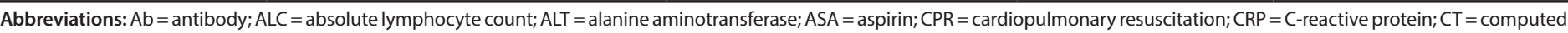

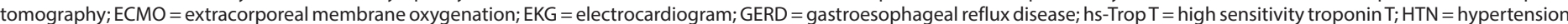

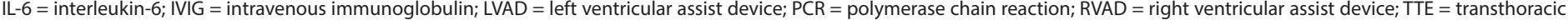
echocardiogram.

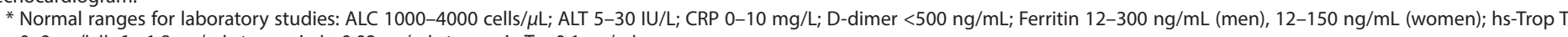
$0-9 \mathrm{ng} / \mathrm{L} \mathrm{IL}-6 \leq 1.8 \mathrm{pg} / \mathrm{mL}$; troponin I $<0.03 \mathrm{ng} / \mathrm{mL}$; troponin $\mathrm{T}<0.1 \mathrm{ng} / \mathrm{mL}$.

† https://www.sciencedirect.com/science/article/pii/S0735675720305428?via\%3Dihub.

$\S$ https://www.thelancet.com/pdfs/journals/lancet/PIIS0140-6736(20)31526-9.pdf.

१ https://www.nejm.org/doi/10.1056/NEJMcpc2004975.

** https://www.sciencedirect.com/science/article/pii/S2665991320302344?via\%3Dihub.

${ }^{\dagger+}$ https://www.acpjournals.org/doi/10.7326/L20-0882.

$\S \S \mathrm{https}: / /$ bmcinfectdis.biomedcentral.com/articles/10.1186/s12879-020-05439-z.

१ๆ https://ard.bmj.com/content/early/2020/09/25/annrheumdis-2020-218959.

syndrome. Additional cases might not have been reported or published; others might have remained unrecognized because of absence of COVID-like symptoms, lack of antibody testing, or negative test results. Second, the working case definition excludes patients with severe respiratory dysfunction to distinguish MIS-A from severe COVID-19; however, the two conditions might overlap in some cases. Finally, the working case definition for this syndrome is potentially nonspecific, and some patients with other disease processes might have been misclassified as having MIS-A.
Clinicians and health departments should consider MIS-A in adults with signs and symptoms compatible with the current working MIS-A case definition. Antibody testing for SARS-CoV-2 might be needed to confirm previous COVID-19 infection in patients who do not have positive SARS-CoV-2 PCR or antigen test results. Findings in this convenience sample emphasize the importance of collecting race/ethnicity data on case reports at the jurisdictional level. As with children, it is important that multidisciplinary care be considered to ensure optimal treatment. In the process of learning more 


\section{Summary}

What is already known about this topic?

Multisystem inflammatory syndrome in children (MIS-C) is a rare but severe complication of SARS-CoV-2 infection in children and adolescents. Since June 2020, several case reports and series have been published reporting a similar multisystem inflammatory syndrome in adults (MIS-A).

What is added by this report?

Cases reported to CDC and published case reports and series identify MIS-A in adults, who usually require intensive care and can have fatal outcomes. Antibody testing was required to identify SARS-CoV-2 infection in approximately one third of 27 cases.

What are the implications for public health practice?

Clinical suspicion and indicated SARS-CoV-2 testing, including antibody testing, might be needed to recognize and treat adults with MIS-A. Further research is needed to understand the pathogenesis and long-term effects of this condition. Ultimately, the recognition of MIS-A reinforces the need for prevention efforts to limit spread of SARS-CoV-2.

from MIS-A cases, the working case definition might need to be revised in order to systematically conduct a call for cases. Further research is needed to understand the pathogenesis and long-term effects of this newly described condition. Ultimately, the recognition of MIS-A reinforces the need for prevention efforts to limit spread of SARS-CoV-2.

\section{Acknowledgments}

Mike Antwi, Robert Atmar, Joshua Beckman, Lucy Bell, William Bender, John Brooks, Cassandra Calabrese, Leonard Calabrese, Eva Clark, Kathy Como-Sabetti, Richard Danila, Kristen Ehresmann, Alisa Femia, Adi Gundapalli, Julie Hoffman, Fanny Ita-Nagy, Elizabeth Jenny-Avital, Ruth Lynfield, Vincent Marconi, Noah Moss, Sarah Reagan-Steiner, Kathleen Reilly, Joshua Scheers-Masters, Julia Schillinger, Ann Schmitz, Kirk Smith, Joanna Taliano, Melissa Tobin-D’Angelo, Richard Vander Heide, Meredith Ventura, Karen Wong.

Corresponding author: Sapna Bamrah Morris, feu3@cdc.gov.

${ }^{1}$ CDC COVID-19 Response Team; ${ }^{2}$ Epidemic Intelligence Service, CDC; ${ }^{3}$ University of Miami Miller School of Medicine and Jackson Health System, Florida; ${ }^{4}$ New York City Department of Health and Mental Hygiene; ${ }^{5}$ Massachusetts General Hospital, Boston, Massachusetts; ${ }^{6}$ Broad Institute of MIT and Harvard, Cambridge, Massachusetts; ${ }^{7}$ Maine Center for Disease Control and Prevention; ${ }^{8}$ University of Southern Maine, Portland, Maine; ${ }^{9}$ Maine Medical Center/Maine Medical Partners, Portland, Maine; ${ }^{10}$ Minnesota Department of Health; ${ }^{11}$ Lousiana State University Health Sciences Center, New Orleans, Louisiana; ${ }^{12}$ Southeast Louisiana Veterans Healthcare System, New Orleans, Louisiana; ${ }^{13}$ Louisiana Department of Health; ${ }^{14}$ Section of Infectious Diseases, Atlanta VA Medical Center, Decatur, Georgia; ${ }^{15}$ Division of Infectious Diseases, Emory University School of Medicine, Atlanta, Georgia;

${ }^{16}$ Oak Ridge Institute for Science and Education, Oak Ridge, Tennessee.
All authors have completed and submitted the International Committee of Medical Journal Editors form for disclosure of potential conflicts of interest. Lilian Abbo reports personal fees from Pfizer, Merck/MSD, Nabriva Therapeutics, Roche Diagnostics, Paratek, and Achaogen, outside the submitted work. Sharon E. Fox reports personal fees from Boehringer Ingelheim, outside the submitted work. Christopher Newton-Cheh reports grants from the National Institutes of Health, and personal fees from GE Healthcare, and Novartis, outside the submitted work. No other potential conflicts of interest were disclosed.

\section{References}

1. Godfred-Cato S, Bryant B, Leung J, et al.; California MIS-C Response Team. COVID-19-associated multisystem inflammatory syndrome in children-United States, March-July 2020. MMWR Morb Mortal Wkly Rep 2020;69:1074-80. https://doi.org/10.15585/mmwr.mm6932e2

2. Belot A, Antona D, Renolleau S, et al. SARS-CoV-2-related paediatric inflammatory multisystem syndrome, an epidemiological study, France, 1 March to 17 May 2020. Euro Surveill 2020;25:2001010. https://doi. org/10.2807/1560-7917.ES.2020.25.22.2001010

3. Whittaker E, Bamford A, Kenny J, et al.; PIMS-TS Study Group and EUCLIDS and PERFORM Consortia. Clinical characteristics of 58 children with a pediatric inflammatory multisystem syndrome temporally associated with SARS-CoV-2. JAMA 2020;324:259-69. https://doi. org/10.1001/jama.2020.10369

4. Chau VQ, Giustino G, Mahmood K, et al. Cardiogenic shock and hyperinflammatory syndrome in young males with COVID-19. Circ Heart Fail 2020. https://doi.org/10.1161/CIRCHEARTFAILURE.120.007485

5. Oxley TJ, Mocco J, Majidi S, et al. Large-vessel stroke as a presenting feature of Covid-19 in the young. N Engl J Med 2020;382:e60. PMID:32343504 https://doi.org/10.1056/NEJMc2009787

6. Magro C, Mulvey JJ, Berlin D, et al. Complement associated microvascular injury and thrombosis in the pathogenesis of severe COVID-19 infection: a report of five cases. Transl Res 2020;220:1-13. https://doi.org/10.1016/j.trsl.2020.04.007

7. Gupta A, Madhavan MV, Sehgal K, et al. Extrapulmonary manifestations of COVID-19. Nat Med 2020;26:1017-32. https://doi.org/10.1038/ s41591-020-0968-3

8. Henderson LA, Canna SW, Friedman KG, et al. American College of Rheumatology clinical guidance for pediatric patients with multisystem inflammatory syndrome in children (MIS-C) associated with SARS-CoV-2 and hyperinflammation in COVID-19. version 1. Arthritis Rheumatol 2020. https://doi.org/10.1002/art.41454

9. Hanson KE, Caliendo AM, Arias CA, et al. Infectious Diseases Society of America guidelines on the diagnosis of COVID-19: serologic testing. Arlington, VA: Infectious Diseases Society of America; 2020. https:// www.idsociety.org/practice-guideline/covid-19-guideline-serology/

10. CDC. Information for healthcare providers about multisystem inflammatory syndrome in children (MIS-C). Atlanta, GA: US Department of Health and Human Services, CDC; 2020. https://www. cdc.gov/mis-c/hcp/ 